\title{
The mystery poor prognosticator! Pharmacologic stress MPI prevalence and predictors: Insights from the Middle East
}

\author{
Mohamed Mandour Ali, MD, ${ }^{a}$ and Adel H. Allam, MD, FASNC ${ }^{a}$ \\ a Cardiology and Cardiovascular Imaging, Al-Azhar University, Cairo, Egypt
}

Received Oct 24, 2017; accepted Oct 24, 2017

doi: 10.1007/s12350-017-1157-8

\section{See related article, pp. 1708-1714}

In this issue of The Journal, Dakik et al. compared the referral patterns of pharmacologic and physical stress myocardial perfusion imaging (MPI) at the American University of Beirut Medical Center (AUMC), a large tertiary hospital serving Lebanon and the Middle East region.

The message from this study would seem to be of significant importance to the daily practice of nuclear cardiology in many developing countries worldwide. The authors concluded that patients referred for pharmacologic stress were older and predominantly female compared to patients undergoing exercise stress. The pharmacologic stress patients also had a higher prevalence of diabetes mellitus and hypertension. The study also showed interesting differences between MPI data from the pharmacologic and physical stress groups. In the pharmacologic stress group, there was a higher prevalence of abnormal scans as well as a higher prevalence of ischemia on MPI and more frequent impaired left ventricular function with an ejection fraction $<50 \%$. The significant predictors for referral to pharmacologic stress were older age and diabetes mellitus. The authors showed similar results on a separate analysis including only patients with no history of prior CAD. They also showed similar results for both genders.

The message coming from this article provokes several important questions and thoughts.

Reprint requests: Adel H. Allam, MD, FASNC, Cardiology and Cardiovascular Imaging, Al-Azhar University, Cairo, Egypt; aallamegy@netscape.net

J Nucl Cardiol 2018;25:1715-7.

$1071-3581 / \$ 34.00$

Copyright (C) 2018 American Society of Nuclear Cardiology.
What are the reasons behind differences in utilization of pharmacologic MPI between developed and developing countries?

The cost and lack of availability of the new pharmacologic stressors represent a challenge to world regions where $\mathrm{CAD}$ mortality is continuously on the rise. How does this affect the availability and utilization of MPI in these regions?

Metabolic syndrome is one of the great health problems in the world right now, particularly in developing countries, and is tightly linked to physical inactivity and sedentary life styles. It represents a link between higher referral to pharmacologic rather than physical exercise, and at the same time is an important direct cause of high-risk myocardial perfusion studies. How can optimal utilization of MPI help in addressing this global critical health problem?

Among the limitations of this study, as stated by the authors, is the relatively small number of patients, and that the study is a single-center investigation that may not necessarily represent the whole country or the region. Also, there was no follow-up data in this study. However, to our knowledge this is the first prospective study comparing referral patterns and predictors of pharmacologic versus physical MPI coming out of the Middle East region.

The robust data over many decades from multiple scientific reports and meta-analysis have proved the clear effectiveness of both physical and pharmacologic MPI studies as diagnostic and prognostic tools in the evaluation of patients with known or suspected CAD. ${ }^{1}$ Moreover, in terms of safety, all pharmacologic stressors used in conjunction with MPI have proved safety margin comparable to treadmill exercise, and to each other. ${ }^{2,3}$

Recent advances in pharmacologic stress agentsthe introduction of adenosine and later regadenosonhave made pharmacologic stress a more convenient choice for patients as well as stress laboratory teams. The short half-life of adenosine and the more selective 
nature and single bolus protocol of regadenoson made pharmacologic stress protocols more suitable, even among higher-risk groups. ${ }^{3}$

Despite these advances, however, physical exercise not only retains significant cost effectiveness, but also offers the advantage of providing an evaluation of the functional capacity of the patient, an important prognostic metric, and one that is closely related to quality of life and well-being of patients. ${ }^{4}$

Bourque et al. concluded that patients who are able to achieve 10 METs on simple treadmill exercise tolerance testing (ETT), without relevant symptoms, ECG changes, and/or hemodynamic compromise, are considered a low-risk group for significant ischemia and cardiac events. They indicated that simple ETT in this group of patients is comparable to MPI in terms of diagnostic accuracy and prognostic power. ${ }^{5}$

There is no doubt that the pattern of patient referral to pharmacologic or physical stress varies from one center to another and is related to multiple factors. From our point of view, the cost (economic factor) and the availability of pharmacologic stressors, along with a patient's cultural background and comorbidities are the main driving factors to the type of stress that is chosen.

Cost and cost effectiveness are leading factors in the referral pattern in many areas. In Egypt, a low- to middle-income Middle Eastern country, the cost of the pharmacologic stressor alone, particularly the newer pharmacological stressors such as regadenoson, may exceed the cost of the whole treadmill MPI test. Of note, regadenoson availability in the Middle East is markedly limited, if available at all. Even adenosine is of limited availability and carries a high cost in Egypt and in many other Middle East countries where dipyridamole is the most widely used pharmacologic stress agent. However, the wide range of side effects, although relatively mild, as well as the relative longer duration of action of dipyridamole compared to newer pharmacologic stressors makes it a subsidiary choice to physical stress MPI.

Dakik and his colleagues in the present study reported that the rate of pharmacologic MPI was less than that reported previously by Rozanski et al. (23\% vs $53 \%$ ). Rozanski reported in his article that the rate of pharmacologic MPI has been steadily increasing in the USA for unclear reasons. He suggested that this change might be due to the increasing sedentary style among the US patients or the convenience of pharmacologic stress. ${ }^{2}$ Dakik et al. believe that one other reason for this apparent difference may be the lower rate of inpatients in their population compared to that of Rozanski ( $8 \%$ vs $43 \%)$.

Similarly, data reported from some of our largest Nuclear Cardiology Labs in Egypt (including university hospitals as well as outpatient radiology centers) show that only $10 \%-30 \%$ of MPI are performed with pharmacological stressors, mainly dipyridamole, a finding that is primarily related to the lower cost of physical exercise and unavailability/shortage of pharmacologic stressors in the Egyptian market.

Surprisingly, unpublished data presented in the last ASNC/IAEA Webinar aired on October 10, 2017 (Nuclear cardiology in the Arab world), by Al-Mallah et al. showed that the pharmacologic stress MPI in Saudi Arabia (a high income Middle Eastern country) approaches $80 \%-90 \%{ }^{6}$

This striking statistic almost certainly reflects the status of physical inactivity in Saudi Arabia which also has a very high prevalence of obesity, metabolic syndrome, and diabetes mellitus. A high incidence of abnormal MPI studies is also seen. ${ }^{6}$ It seems clear that the varying referral pattern is neither related to geography nor to special expertise. It is mainly related to cost, availability of pharmacologic stressor, and to patients comorbidities.

Data presented in 2004 by Navare et al. are highly relevant. This study is a meta-analysis in 14819 patients and reported that exercise stress MPI and pharmacologic stress MPI are comparable in their ability to risk-stratify patients. However, patients undergoing pharmacologic stress studies tend to be a sicker group by the fact that they are not able to exercise, and are at a higher risk for subsequent cardiac events, even when the MPI test is normal. ${ }^{7}$ Rozanski et al. reported similar findings among patients tested with exercise or adenosine stress protocol. ${ }^{8}$

Dakik et al. also reported that, in spite of the decline in the rate of abnormal or ischemic MPI that has been reported in the USA and in the Middle East region over the last two decades, the rates of ischemia at the American University of Beirut Medical Center remain relatively high., ${ }^{2,9}$ Indeed, the rates of abnormal or ischemic MPI in their population remain higher than that reported by Rozanski during the same time period. Dakik et al. cautioned that this difference might be secondary to the higher rates of diabetes and smoking among patients in the Middle East. In our opinion, this demonstration of high rates of ischemia is one of the important findings in the current study; it is not only a relevant nuclear cardiology issue, but also speaks to common public health problems that should be thoroughly investigated and managed, especially in regions with limited resources. We also believe that the findings reported in the current study by Dakik et al. should alarm local health authorities. There are increasing rates of physical inactivity (and subsequently the metabolic syndrome) leading to referral to pharmacologic rather than physical exercise stress testing, and related to higher-risk MPI studies with likely subsequent higher rates of morbidity and mortality in these patients. 


\section{References}

1. Shaw LJ, Hendel R, Borges-Neto S, et al. Prognostic value of normal exercise and adenosine $(99 \mathrm{~m}) \mathrm{Tc}$-tetrofosmin SPECT imaging: Results from the multicenter registry of 4728 patients. J Nucl Med 2003;44:134-9.

2. Rozanski A, Gransar H, Hayes SW, Min J, Friedman JD, Thomson LE, et al. Temporal trends in the frequency of inducible myocardial ischemia during cardiac stress testing: 1991 to 2009. J Am Coll Cardiol. 2013;61:1054-65.

3. Dilsizian V, Gewirtz H, Paivanas N, et al. Serious and potentially life threatening complications of cardiac stress testing: physiological mechanisms and management strategies. J Nucl Cardiol 2015;22:198-13.

4. Fagan LF Jr, Shaw L, Kong BA, et al. Prognostic value of exercise thallium scintigraphy in patients with good exercise tolerance and a normal or abnormal exercise electrocardiogram and suspected or confirmed coronary disease. Am J Cardiol 1992;69:607-11.
5. Bourque JM, Charlton GT, Holland BH, et al. Prognosis in patients achieving $\geq 10$ METS on exercise stress testing: was SPECT imaging useful? J Nucl Cardiol. 2011;18:230-7.

6. Al-Mallah M, Allam AH (2017) Nuclear cardiology in the Arab world. ASNC/IAEA Webinar aired on October 10, 2017.

7. Navare MS. MD, Mather FJ, Shaw JL, Fowler SM and Heller VG. Comparison of risk stratification with pharmacologic and exercise stress myocardial perfusion imaging: a meta-analysis. J Nucl Cardiol. 2004;11:551-61.

8. Rozanski A, Gransar H, Hayes WS, Friedman DJ, Hachamovitch R, Berman SD. Comparison of long-term mortality risk following normal exercise vs adenosine myocardial perfusion SPECT. J Nucl Cardiol. 2010;17:999-1008.

9. Thomspon R, Allam AH. More risk factors, less ischemia, and the relevance of MPI testing. J Nucl Cardiol. 2015;22:552-4. 